\title{
Quadratic Approximation of the Newsvendor Problem with Imperfect Quality
}

\author{
Natapat Areerakulkan* \\ Department of Engineering Management, College of Innovative Technology and Engineering, Dhurakij Pundit \\ University, Bangkok, Thailand
}

\begin{abstract}
The paper presents a newsvendor problem in a fuzzy environment by introducing product quality as a fuzzy variable, and product demand as a probability distribution in an economic and supply chain management environment. In order to determine the optimal order quantity, a methodology is developed where the solution is achieved using a fuzzy ranking method combined with a quadratic programming problem approximation. Numerical examples are provided and compared in both situations, namely fuzzy and crisp. The results of these numerical examples show that the decision maker has to order a higher quantity when product quality is a fuzzy variable. The model can be useful for real world problems when historical data are not available.
\end{abstract}

Keywords: Imperfect quality items, Newsvendor problem, Quadratic programming.

\section{INTRODUCTION}

The classical newsvendor problem has been developed for perishable products with limited lifetime. Various types of perishable products, such as newspaper and magazine, flowers, fresh food, fresh vegetable, have additional costs of disposal. The two other basic cost components related to the newsvendor problem are the inventory holding cost and opportunity cost. The inventory holding cost is incurred when a seller orders more than can be sold. The loss of opportunity cost is incurred when the order is less than can be sold. When all cost components are combined, they play an important role in business costs that need to be minimized for surviving in highly competitive environment. Therefore, it is important to determine the correct order quantity match to customer demand, and hence the maximum profit or minimum cost.

The newsvendor problem has become increasingly interested as illustrated by several papers published since 1988 and covering various extensions. The relevant studies are explained as follows. Lau and Lau $(1995,1996)$ developed a simple algorithm for solving the multi-product constrained newsvendor model. They also mentioned that the solution might be a negative order quantity when the budget constraint is tight. Ben-Daya and Raouf (1993) and Erlebacher (2000) introduced the optimal and heuristic solution method for the newsboy problem with one constraint. Niederhoff (2007) introduced a linear programming formulation for the multi-constraint newsvendor problem where the objective function is approximated and optimized by linear segments. Abdel-Malek and Areeratchakul (2007) developed a quadratic

*Address correspondence to this author at the Department of Engineering Management, College of Innovative Technology and Engineering, Dhurakij Pundit University, Bangkok, Thailand; Tel: +66(2) 9547300; Fax: +66(2) 9547356; E-mail: natapat.arn@dpu.ac.th

JEL: C61, C63, L15. programming approach for solving the multi-constraint problem, and uses familiar software packages such as Excel.

Khouja (1999) classified the newsvendor problem into 11 categories, namely extensions to; different objectives and utility functions; different supplier pricing policies; different pricing policies and discount structures; random yield; different states of information about demand; constrained multi-product; multi-product with substitution; multi-echelon systems; multi-location models; and models with more than one period. Choi (2012) also consolidated the state-of-the-art findings from more than one thousand papers related to the problem (see the Handbook of Newsvendor Problems).

As previously mentioned, the current literature of the classical newsvendor problem are immensely diversified. However, this research is interested in the more practical extension, which is the imperfect quality problem. This problem occurs due to errors in the production process, natural disasters, and damage in transit, among others. As a result, the order quantity contains defective products rather than perfect quality, as considered in the traditional newsboy problem. It is an important problem, and is generally realized in real world applications, which can be characterized in many production systems, such as agriculture in the production of fruits or vegetables, production of computer processors, and production of chemicals. For example, in semiconductor manufacturing, it is possible to face yield losses which can exceed $80 \%$, as mentioned in Nahmias (1997).

In the fast growing remanufacturing industry, the disassembly processes face, with high quality rate fluctuations stem from the limited knowledge of the quality of used products. Other than in the production system, in procurement processes, we can face quality uncertainty derived from unreliable delivery quantities 
of suppliers. Imperfect quality is an important problem. Despite the importance of the problem, insufficient attention has been given to this problem when considering multi-items with limited resources (Abdel-Malek et al., 2008).

The literature regarding the imperfect quality items case can be categorized into two scenarios, namely: 1) the newsvendor problem with imperfect quality denoted by a probability distribution, called the random yield scenario; and 2) the imperfect quality, denoted by a fuzzy variable is the purpose of the paper.

For the random yield scenario, several papers focus on the stochastically proportional yield, which assumes that the fraction of good units is a random variable independent of the batch size. This type of yield is suitable for the production environment where batch sizes are relatively large. For further details, see Gerchak et al. (1998) and Henig and Gerchak (1990), where it is shown that the optimal order quantity is not a linear function of the inventory level.

Ehrhardt and Taube (1987) derived closed-form solutions for the problem when demand and yield distributions have a uniform distribution. Lee and Yano (1988) provided a comprehensive review of the lot sizing problem with random yields. Noori and Keller (1986) derived the optimal solution order quantity for the unconstrained newsvendor problem with random yield for both random demand and yield. Inderfurth (2004) derived the optimal solution for the single item newsvendor problem for uniformly distributed demand and yield. Abdel-Malek et al. (2008) extended their work from that of Abdel-Malek and Montanari (2004) to cover the random yield case. They assumed that the decision variable (the amount to be planted) is the upper bound of yield, and the starting inventory is zero. They developed an algorithm based on an iterative process to solve the multi-item constrained problem for general distributed demand and yield.

As previously mentioned, some research considers the fuzzy yield. However, there are several works related to the newsvendor problem with fuzzy demand and additional extended parameter, as summarized in Table 1

Greater detail of the literature on the newsvendor problem in a fuzzy environment is explained as follows. $\mathrm{Yu}$ et al. (2013) propose a newsvendor model with fuzzy price-dependent demand. The conditions to determine optimal pricing is proposed. The effect of the uncertain price-dependent demand is studied by comparing the fuzzy and deterministic case. Chen and Ho (2011) study the inventory problem with fuzzy demands and incremental quantity discounts. The Yager ranking method is implemented to transform fuzzy total cost functions into convex piecewise nonlinear functions. Closed-form solutions to the optimal order quantities are also derived. $\mathrm{Xu}$ and $\mathrm{Hu}$ (2012) develop the profit maximized newsboy model with random fuzzy demand, and use credibility measure of the fuzzy event to obtain expected profit model. A hybrid algorithm integrating the random fuzzy simulation and simultaneous perturbation stochastic approximation is developed to obtain the optimal order quantity.

Taleizadeh et al. (2010) consider the newsvendor problem with demand of each product as LR-fuzzy variables and multiple constraints. Algorithms such as bees colony optimization (BCO), harmony search (HS), particle swarm optimization (PSO), genetic algorithm

Table 1: Newsvendor Problem in Fuzzy Environment

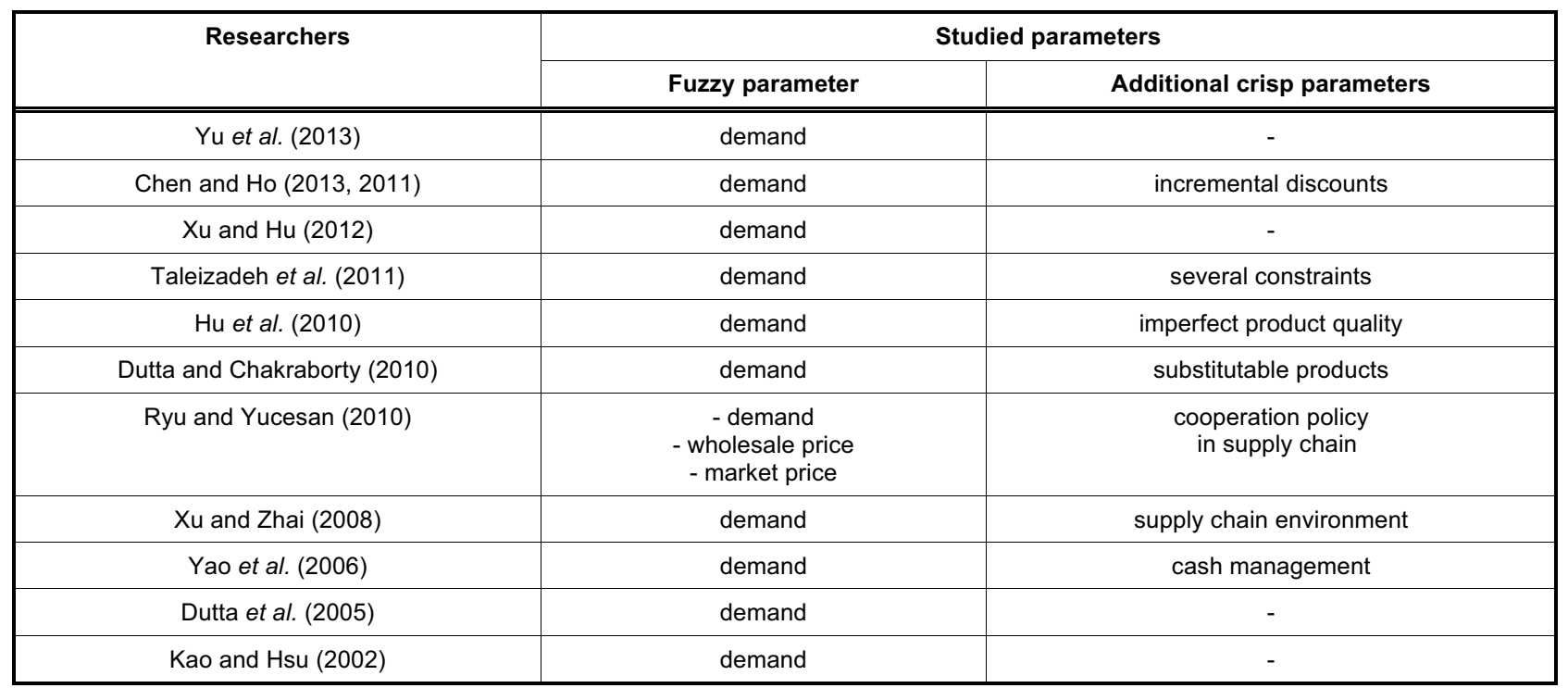


(GA) and simulated annealing (SA), are implemented to obtain order quantiy that maximizes profits. Hu et al. (2010) consider the newsvendor problem with fuzzy random demand and imperfect quality (random yield) in a two-echelon supply chain system. The optimal policies are derived and analyzed. Dutta and Chakraborty (2010) consider the problem with fuzzy demand and one way substitutable products. The optimal order quantities and average expected profit under substitution are provided. Ryu and Yucesan (2010) solve the fuzzy newsvendor problem in the supply chain management arena to study three coordination policies, namely quantity discounts, profit sharing, and buyback. They use several fuzzy parameters in the model which are demand, wholesale price, and market sales price. For each coordination policy, the optimal order quantity of the retailer is computed.

$\mathrm{Xu}$ and Zhai (2008) consider a single-period problem with the fuzziness aspect of demand uncertainties. Triangular fuzzy numbers are used for external demand. Two decision models are developed, which are non-coordination and coordination. The analytical solution is then calculated and expressed. Yao et al. (2006) apply a stochastic single-period inventory management approach to analyze optimal cash management policies, with fuzzy cash demand based on the fuzzy integral method. The objective function is to minimize total cost. Their results show that the cash-raising amounts and total costs between the fuzzy and the crisp cases are slightly different. Dutta et al. (2005) present a single-period inventory problem when demand is a fuzzy random variable. The optimal order quantity is developed using a graded mean integration representation. Kao and Hsu (2002) consider single-period inventory models with fuzzy demand. The method for ranking fuzzy numbers is adopted to find the optimal order quantity that minimizes total cost.

From the literature review, one can see that the previous work focuses on the single item uncapacitated fuzzy demand problem. Insufficient attention is focused on both the fuzzy quality rate parameter and the probability distribution demand for multi-item with capacitated constraints problem. Therefore, we extend the problem to consider the situation when there is no historical data on the quality of items, or the ordered products are prone to damage before reaching customers. As previously mentioned, this kind of situation is well written and reported for the semiconductor products. The outcome is the uncertainty of the quality of product, which can cause difficulties for decision makers regarding the quantity of perfect quality product they should receive. Therefore, to cope with this problem we represent the uncertainty with a fuzzy number as the fuzzy quality rate. Furthermore, we have to deal with another uncertainty represented by the probability distribution function, namely customer demand, which makes the problem more complex.

The methodology is based on two main steps, which are the quadratic approximation of the objective function and iterative solution finding steps using the KKT's condition method. Additionally, numerical examples are provided to illustrate the application of the model.

This paper is organized as follows. Introduction and literature review are given in section 1 . Sections 2, 3, and 4 present the model and the necessary preliminaries. Section 5 illustrates the numerical example for different demand distributions. The conclusions are given in Section 6 .

\section{FUZZY QUALITY RATE}

Fuzzy product quality can occur in several situations, such as: 1) when the product is easy to damage during the transportation; and 2) when the product is a new product with no historical quality related data. The outcome can cause uncertainty in the received quantity, for example, ordering 100 units but receiving only 80 good units which might not fulfill the customer order. In this research, we represent this uncertainty by using a fuzzy number as the following equation:

$\tilde{Q}_{\tau}=\tilde{\varphi}_{\tau} x_{\tau}$

where $\tilde{\varphi}_{\tau}=\left(1-\tilde{\gamma}_{\tau}\right), \quad \tilde{Q}$ is denoted as the fuzzy quantity received, $\varphi_{\tau}$ is denoted as the fuzzy quality rate, $\tilde{\gamma}_{\tau}$ is denoted as the fuzzy defective rate, $x_{\tau}$ is denoted as the quatity to be ordered.

Suppose that $\tilde{\gamma}_{\tau}=\left(l_{\tau}, m_{\tau}, n_{\tau}\right)$ is a triangular fuzzy number described by the following membership function:

$\mu_{\widetilde{\gamma}_{\tau}}(q)= \begin{cases}L_{\tau}(q)=\left(\frac{q-l_{\tau}}{m_{\tau}-l_{\tau}}\right), l_{\tau} \leq q \leq m_{\tau}, \\ R_{\tau}(q)=\left(\frac{n_{\tau}-q}{n_{\tau}-m_{\tau}}\right), & m_{\tau} \leq q \leq n_{\tau}, \\ 0, & \text { otherwise. }\end{cases}$

In the next section, the model is developed and explained.

\section{FUZZY QUALITY MODEL FOR THE NEWSVENDOR PROBLEM}

Consider a newsvendor problem with fuzzy quality items. Due to the lack of historical data, the quality of each item is estimated by the experts depending on their experience and managerial subjective judgment. Therefore, these characteristics are better described by 
the use of fuzzy sets which encompass a specific range of values.

The fuzzy product quality newsvendor problem is given, as follows:

$\operatorname{Min} \widetilde{T C}\left(D_{\tau}, \widetilde{Q}_{\tau}\right)=c_{\tau} \widetilde{Q}_{\tau}+h_{\tau} \max \left(0, \widetilde{Q}_{\tau}-D_{\tau}\right)+$ $v_{\tau} \max \left(0, D-\widetilde{Q}_{\tau}\right)$.

By substituting $\tilde{Q}_{\tau}=\left(1-\tilde{\gamma}_{\tau}\right) x_{\tau}$ into equation (3) we obtain:

$\operatorname{Min} \widetilde{T C}\left(D_{\tau}, \widetilde{Q}_{\tau}\right)=c_{\tau}\left(1-\tilde{\gamma}_{\tau}\right) x_{\tau}+h_{\tau} \max \left(0,\left(1-\tilde{\gamma}_{\tau}\right) x_{\tau}-\right.$ $\left.D_{\tau}\right)+v_{\tau} \max \left(0, D-\left(1-\tilde{\gamma}_{\tau}\right) x_{\tau}\right)$,

subject to:

$\sum_{\tau=1}^{N}\left(c_{\tau} x_{\tau}\right) \leq B_{g}$

$\sum_{i=1}^{M} \sum_{\tau=1}^{N}\left(\rho_{i, \tau} x_{\tau}\right) \leq R_{i}, i=1,2, \ldots, M$,

$\forall_{\tau=1}^{N} x_{\tau} \geq 0$

where $\tau$ is item type, $D_{\tau}$ is demand, $x_{\tau}$ is order quantity, $c_{\tau}$ is unit cost, $h_{\tau}$ is holding cost, $v_{\tau}$ is the loss of opportunity cost, $\mathrm{Bg}$ is the available budget, $\tilde{\gamma}_{\tau}$ is the fuzzy defective rate, $\rho_{i, \tau}$ is unit shelf space required, and $R_{i}$ is available space. After obtaining the model, the solution method is developed, and the details are given in the following section.

\section{SOLUTION METHODOLOGY}

The first step of the methodology is to transform the fuzzy objective function into the quadratic function. The detail of this step is mentioned as follows. As in the previous section, total cost $(\widetilde{T C})$ is fuzzy number. Thus, it can be ranked by fuzzy ranking methods to find the optimal order quantity. In the literature, several ranking methods have been proposed and discussed. Most of the methods require knowledge of the membership functions of the fuzzy number to be ranked.

The method of Yager (1981) does not need to know the explicit form of the membership functions, and can be defined as:

$I\left(\widetilde{T C}_{\tau}(\alpha)\right)=\int_{0}^{1} \frac{1}{2}\left(T C_{\tau}^{L}+T C_{\tau}^{R}\right) d \alpha$

where $\widetilde{T C}_{\tau}(\alpha)$ represents the $\alpha$-cut, or $\alpha$-level set of the total cost, $T C_{\tau}^{L}$ represents the area bounded by the left shape function of the total cost (see equation (6)), and $T C_{\tau}^{R}$ represents the area bounded by the right shape function of the total cost (see equation (7)),

As demand is a probability random distribution, we must implement its expected value as the following equation (Xu and $\mathrm{Hu}, 2012$ ):

$I\left(E\left[\widetilde{T C_{\tau}}, \alpha\right]\right)=\int_{0}^{1} \frac{1}{2}\left(E\left[T C_{\tau}^{L}\right]+E\left[T C_{\tau}^{R}\right]\right) d \alpha$,
$E\left[T C_{\tau}^{L}\right]=\left(c_{\tau}-v_{\tau}\right)\left(1-L_{\tau}^{-1}(\alpha)\right) x_{\tau}-$

$\left(h_{\tau}+v_{\tau}\right) \int_{0}^{\left(1-L_{\tau}^{-1}(\alpha)\right) x_{\tau}} F\left(D_{\tau}\right) d D_{\tau}-v_{\tau} E\left[D_{\tau}\right]$,

$l_{\tau} \leq q \leq m_{\tau}$

$E\left[T C_{\tau}^{R}\right]=\left(c_{\tau}-v_{\tau}\right)\left(1-R_{\tau}^{-1}(\alpha)\right) x_{\tau}$

$-\left(h_{\tau}+v_{\tau}\right) \int_{0}^{\left(1-R_{\tau}^{-1}(\alpha)\right) x_{\tau}} F\left(D_{\tau}\right) d D_{\tau}-v_{\tau} E\left[D_{\tau}\right]$,

$m_{\tau} \leq q \leq n_{\tau}$

$L_{\tau}^{-1}(\alpha)=\alpha_{\tau}\left(m_{\tau}-l_{\tau}\right)+l_{\tau}$,

$R_{\tau}^{-1}(\alpha)=n_{\tau}-\alpha_{\tau}\left(n_{\tau}-m_{\tau}\right)$.

After obtaining the objective function as mentioned in equation (5), we present the solution methodology for several scenarios descending from the simple to the complex. These are: 1) one item with no constraint with uniform and general probability distributed demand (sections 4.1 and 4.2); and 2) multi-item with resourced constraints with general probability distributed demand (section 4.3). Then the numerical examples are provided for each scenario.

\subsection{Uniformly Distributed Demand}

Consider one item with no side constraint, then extend the model to cover multi-items with side constraints. For a uniformly distributed product demand, we can represent the demand cumulative distribution fuction and its expected value as equations (10) and (11), respectively:

$F(D)=(D-a) /(b-a)$,

$E(D)=(b+a) / 2$.

Then we substitute equation (6) into equations (11) and (5). We obtain the average total cost function, as follows:

$$
\begin{gathered}
I(T C)=0.5\left\{\left[\frac{-(v+h)\left(2 m^{2}+m(l+n)+n^{2}+l^{2}\right)}{6\left(b_{\tau}-a_{\tau}\right)}\right] x^{2}\right. \\
+\left[\left[\left(\frac{v-c}{2}\right)+a \frac{(v+h)}{2(b-a)}\right](2 m+l+n)\right] x \\
\left.+\frac{\left[-(v+h) a^{2}-v\left(b^{2}-a^{2}\right)\right]}{(b-a)}\right\} .
\end{gathered}
$$

Therefore, without constraints, the optimal order quantity for each item can easily be obtained by $\frac{\partial I(E[T C])}{\partial x}=0$, and then solving to find $x^{*}$. The exact optimal solution is:

$x^{*}=\frac{-0.5\left(\left[\left(\frac{v-c}{2}\right)+a \frac{(v+h)}{2(b-a)}\right](2 m+l+n)\right)}{\left[\frac{-(v+h)\left(2 m^{2}+m(l+n)+n^{2}+l^{2}\right)}{6\left(b_{\tau}-a_{\tau}\right)}\right]}$. 


\subsection{General Distributed Demand}

For general distributed demand, the exact solution cannot be obtained due to the complexity in integration. Thus, we must approximate the solution by using the algorithm developed by Areeratchakul and Abdel-Malek (2006). Applied to this problem, we can approximate equation (5), as follows:

$$
\begin{array}{r}
I(T C)=0.5\left\{\left[\frac{-(v+h)\left(2 m^{2}+m(l+n)+n^{2}+l^{2}\right)}{6}\right] x^{2}\right. \\
+\left[\left[\left(\frac{v-c}{2}\right)+\frac{\Delta k(v+h)}{2}\right](2 m+l\right. \\
\left.+n)] x+\left[-(v+h) \Delta k^{2}-2 v E[D]\right]\right\} .
\end{array}
$$

For the exponentially distributed demand, $\Delta=\frac{\left(1-e^{\frac{-x^{*}}{\mu}}\right)}{x^{*}}, k=0$, while for Normally distributed demand, $\Delta=f(\mu)$, and $k=\mu-\frac{F(\mu)}{f(\mu)}$. Therefore, without considering constraints, the optimal order quantity for each item can easily be obtained by $\frac{\partial I(T C)}{\partial x}=0$, and then solving to find $x^{*}$. The exact optimal solution is given as:

$x^{*}=\frac{-0.5\left(\left[\left(\frac{v-c}{2}\right)+\frac{\Delta k(v+h)}{2}\right](2 m+l+n)\right)}{\left[\frac{-(v+h)\left(2 m^{2}+m(l+n)+n^{2}+l^{2}\right)}{6}\right]}$.

We can represent the $I(T C)$ as a quadratic function, as follows:

$I(T C)=0.5\left[A x^{2}+B x+C\right]$,

where

$$
\begin{aligned}
& A=\left[\frac{-(v+h)\left(2 m^{2}+m(l+n)+n^{2}+l^{2}\right)}{6}\right], \\
& B=\left[\left(\frac{v-c}{2}\right)+\frac{\Delta k(v+h)}{2}\right](2 m+l+n), \\
& C=\left[-(v+h) \Delta k^{2}-2 v E[D]\right] .
\end{aligned}
$$

\subsection{Multi-Items with Side Constraints}

As the newsvendor problem assumes that all items are independent, for the muti-items case, we can calculate the optimal ordered quantity of each item separately. However, when including side constraints, the problem is more complex. As the research approximates the total cost function as a quadratic function, to obtain the solution we could implement the Karush-Khun-Tucker (KKT) conditions. Another less burden some measure to solve the problem when including several items and constraints is to use available software tools for solving optimization models, such as Lindo or Microsoft Excel Solver. The model for multi-items with side constraints for general distributed demand can be represented as follows:
$I(T C)$

$$
\begin{aligned}
& =0.5 \sum_{\tau=1}^{N}\left\{\left[\frac{-\left(v_{\tau}+h_{\tau}\right)\left(2 m_{\tau}^{2}+m_{\tau}\left(l_{\tau}+n_{\tau}\right)+n_{\tau}^{2}+l_{\tau}^{2}\right)}{6}\right] x_{\tau}^{2}\right. \\
& +\left[\left[\left(\frac{v_{\tau}-c_{\tau}}{2}\right)+\frac{\Delta_{\tau} k_{\tau}\left(v_{\tau}+h_{\tau}\right)}{2}\right]\left(2 m_{\tau}+l_{\tau}+n_{\tau}\right)\right] x_{\tau} \\
& \left.+\left[-\left(v_{\tau}+h_{\tau}\right) \Delta_{\tau} k_{\tau}^{2}-2 v_{\tau} E\left[D_{\tau}\right]\right]\right\},
\end{aligned}
$$

subject to

$$
\begin{aligned}
& \sum_{\tau=1}^{N}\left(c_{\tau} x_{\tau}\right) \leq B_{g}, \\
& \sum_{i=1}^{M} \sum_{\tau=1}^{N}\left(\rho_{i, \tau} x_{\tau}\right) \leq R_{i}, i=1,2, \ldots, M,
\end{aligned}
$$

$\forall_{\tau=1}^{N} x_{\tau} \geq 0$

As in the previous section, we can represent $I(T C)$ as a quadratic function, as follows:

$I(T C)=0.5 \sum_{\tau=1}^{N}\left[A_{\tau} x_{\tau}^{2}+B_{\tau} x_{\tau}+C_{\tau}\right]$,

where

$A_{\tau}=\left[\frac{-\left(v_{\tau}+h_{\tau}\right)\left(2 m_{\tau}^{2}+m_{\tau}\left(l_{\tau}+n_{\tau}\right)+n_{\tau}^{2}+l_{\tau}^{2}\right)}{6}\right]$,

$B_{\tau}=\left[\left[\left(\frac{v_{\tau}-c_{\tau}}{2}\right)+\frac{\Delta_{\tau} k_{\tau}\left(v_{\tau}+h_{\tau}\right)}{2}\right]\left(2 m_{\tau}+l_{\tau}+n_{\tau}\right)\right]$,

$C_{\tau}=\left[-\left(v_{\tau}+h_{\tau}\right) \Delta_{\tau} k_{\tau}^{2}-2 v_{\tau} E\left[D_{\tau}\right]\right]$.

In order to solve the Quadratic programming (QP) problem, the KKT conditions can be implemented, as follows:

$\operatorname{Max} f(x)=\omega x-\frac{1}{2} x^{T} Q x$,

subject to

$\Lambda x \leq b$ and $x \geq 0$.

For a QP problem, the KKT conditions can be represented as linear programming constraints, as follows:

$$
\begin{aligned}
& Q x+\wedge^{T} u-y=\omega^{T}, \\
& \wedge x+v=b, \\
& \quad x \geq 0, u \geq 0, y \geq 0 . v \geq 0 \\
& x^{T} y+u^{T} v=0 .
\end{aligned}
$$

Further details are given in Abdel Malek and Areeratchakul (2007). 


\section{NUMERICAL EXAMPLE}

The first example considers only 1 item with no constraint, where the numerical data are given as: $v=25, h=1.6, c=8, a=50, b=200, l=0.5, m=$ $0.7, n=1$. The optimal solution and cost are calculated and compared for the crisp and fuzzy scenarios, as shown in Table 2.

Table 2: Numerical Data

\begin{tabular}{|c|c|c|c|}
\hline \multicolumn{2}{|c|}{ Crisp } & \multicolumn{2}{c|}{ Fuzzy } \\
\hline \hline$A$ & -0.089 & $A$ & -0.097 \\
\hline$B$ & 25.867 & $B$ & 37.507 \\
\hline$C$ & -3346.667 & $C$ & -6693.334 \\
\hline$x^{*}$ & 145.86 & $x^{*}$ & 193.45 \\
\hline$T C$ & 1460.15 & $T C$ & 1532.76 \\
\hline
\end{tabular}

In order to verify the model, let $(I, m, n)$ be $(1,1,1)$, so the optimal solution should be the same as in the crisp scenario. The optimal solution is calculated as $\left(\mathrm{q}^{*}\right.$, $\mathrm{TC})=(145.86,1460.15)$. When the item quality is fuzzy, the decision maker has to order more to cope with uncertainty that might cause undesirable incidents.

The second example extends that of Lau and Lau [1, 2], where all three demands are normally distributed, and we then add the value of $(I, m, n)$ to cover the fuzzy item quality aspect. The numerical data is shown in Table 3

Table 3: Numerical Data

\begin{tabular}{|c|c|c|c|c|c|c|c|}
\hline Item & $\boldsymbol{v}_{\boldsymbol{\tau}}$ & $\boldsymbol{h}_{\boldsymbol{\tau}}$ & $\boldsymbol{c}_{\boldsymbol{\tau}}$ & $\boldsymbol{\mu}_{\boldsymbol{\tau}}$ & $\boldsymbol{l}_{\boldsymbol{\tau}}$ & $\boldsymbol{m}_{\boldsymbol{\tau}}$ & $\boldsymbol{n}_{\boldsymbol{\tau}}$ \\
\hline \hline 1 & 4 & 1 & 0 & 100 & 0.8 & 0.9 & 1 \\
\hline 2 & 1 & 1 & 0 & 500 & 0.7 & 0.9 & 1 \\
\hline 3 & 2 & 2 & 0 & 300 & 0.6 & 0.8 & 0.9 \\
\hline
\end{tabular}

All the required parameters are calculated, as shown in Table 4.

Table 4: Quadratic Function Coefficients

\begin{tabular}{|c|c|c|c|c|}
\hline$\Delta_{\boldsymbol{\tau}}$ & $\boldsymbol{k}_{\boldsymbol{\tau}}$ & $\boldsymbol{A}_{\boldsymbol{\tau}}$ & $\boldsymbol{B}_{\boldsymbol{\tau}}$ & $\boldsymbol{C}_{\boldsymbol{\tau}}$ \\
\hline \hline 0.00497 & 0 & -0.020 & 7.200 & -800 \\
\hline 0.00144 & 0 & -0.002 & 1.750 & -1000 \\
\hline 0.0024 & 0 & -0.006 & 3.100 & -1200 \\
\hline
\end{tabular}

The optimal solution is obtained as $x_{1}^{*}=178.09, x_{2}^{*}$ $=392.14$, and $x_{3}^{*}=264.92$. In a similar manner, to verify the model we compare the result to that of Lau and Lau (1996), which is $x_{1}^{*}=160.9, x_{2}^{*}=346.6$, and $x_{3}^{*}=207.9$. For crisp scenario $\left(l_{\tau}=1, m_{\tau}=1, n_{\tau}=1\right)$, our optimal solution is $x_{1}^{*}=160.9, x_{2}^{*}=346.6$, and $x_{3}^{*}$ $=207.9$, which is identical.

\section{CONCLUSION}

The newsvendor problem has extensive applications in solving real world problems. From the literature survey, there are some papers dealing with both probabilistic demand and fuzzy quality item, which is the main focus of the paper. The newsvendor model is formulated for this situation. Then the solution method is developed by approximating the total cost function, represented as Quadratic function, and using KKT conditions to solve the problem. For the multi-product with side constraints, Microsoft Excel Solver can be implemented.

From the numerical example, we found that the order quantity in the fuzzy environment is more than that of the normal environment. However, we obtain the same solution when the fuzzy parameter is given a value equal to one. As the products are prone to damage and do not have historical data to construct the probability function for calculation, the fuzzy model constructed in this paper should be helpful for solving real world problems.

The author wishes to thank Chia-Lin Chang and Michael McAleer for helpful comments and suggestions. This research was partially supported by Thailand Research Fund under grant MRG5180149.

\section{REFERENCES}

Abdel-Malek L., Areeratchakul N. (2007), A quadratic programming approach to the multi-product newsvendor problem with side constraints, European Journal of Operational Research, 176(3), 1607- 1619. https://doi.org/10.1016/j.ejor.2005.11.002

Abdel-Malek L., Montanari R., Diego M. (2008), The capacitated newsboy problem with random yield: The gardener, International Journal of Production Economics, 115, 113127.

https://doi.org/10.1016/j.ijpe.2008.05.003

Abdel-Malek L., Montanari R. (2004), An analysis of multi-product newsboy problem with a budget constraint, International Journal of Production Economics, 97(3), 296-307. https://doi.org/10.1016/j.ijpe.2004.08.008

Areeratchakul N., Abdel-Malek L. (2006), An approach for solving the multi-product newsboy problem, International Journal of Operational Research, 3, 219-227.

Ben-Daya M., Raouf A. (1993), On the constrained multi-item single period inventory problem, International Journal of Operations \& Production Management Bradford, 13, 104-112. https://doi.org/10.1108/01443579310046472

Chen S. P., Ho Y. H. (2011), Analysis of the newsboy problem with fuzzy demands and incremental discounts. International Journal of Production Economics, 129, 169-177. https://doi.org/10.1016/j.ijpe.2010.09.014

Choi. T. M. (2012), Handbook of Newsvendor Problems: Models, Extensions and Application. Springer, New York. https://doi.org/10.1007/978-1-4614-3600-3

Dutta P., Chakraborty D. (2010), Incorporating one-way substitution policy into the newsboy problem with imprecise customer demand. European Journal of Operational Research, 200, 99-110. https://doi.org/10.1016/j.ejor.2008.12.011 
Dutta P., Chakraborty D., Roy A. R. (2005), A single-period inventory model with fuzzy random variable demand, Mathematical and Computer Modelling, 41, 915-922. https://doi.org/10.1016/j.mcm.2004.08.007

Erlebacher S.J. (2000), Optimal and heuristic solutions for the multi-item newsvendor problem with a single capacity constraint, Production and Operations Management, 9(3), 303-318. https://doi.org/10.1111/j.1937-5956.2000.tb00139.x

Ehrhardt R., Taube L. (1987), An inventory model with random replenishment quantities, Journal of Production Research, 25(12), 1795-1803.

Gerchak Y., Vickson R.G., Parlar M. (1988), Periodic review production models with variable yield and uncertain demand, IIE Transactions, 20(2), 144-150. https://doi.org/10.1080/07408178808966163

Henig M., Gerchak Y. (1990), The structure of periodic review policies in the presence of variable yield, Operations Research, 38, 634-643. https://doi.org/10.1287/opre.38.4.634

Ji Y. P., Guo C. Y. (2010), Supply chain coordination for fuzzy random newsboy problem with imperfect quality, International Journal of Approximate Reasoning, 51(7), 771-784.

Inderfurth K. (2004), Analytical solution for a single-period production-inventory problem with uniformly distributed yield and demand, Central European Journal of Operations Research, 12(2), 117-127.

Kao C., Hsu W. K. (2002), A single-period inventory model with fuzzy demand, Computer and Mathematics with Application, 42, 841-848. https://doi.org/10.1016/S0898-1221(01)00325-X

Khouja M. (1999), The single-period (news-vendor) problem: Literature review and suggestions for future research, Omega International Journal of Management Science, 27, 537-553. https://doi.org/10.1016/S0305-0483(99)00017-1

Lau H. S., Lau A. H. L. (1995), The multi-product multi-constraint Newsboy problem: Applications formulation and solution, Journal of Operations Management, 13, 153-162. https://doi.org/10.1016/0272-6963(95)00019-O

Lau H. S., Lau A. H. L. (1996), The newsstand problem: A capacitated multiple-product single period inventory problem, European Journal of Operational Research, 94, 29-42. https://doi.org/10.1016/0377-2217(95)00192-1
Lee H.L., Yano C.A. (1988), Production control in multistage systems with variable yield losses. Operations Research, 36(2), 269-278. https://doi.org/10.1287/opre.36.2.269

Nahmias S., Moinzadeh K. (1997), Lot sizing with randomly graded yields, Operations Research, 974-989. https://doi.org/10.1287/opre.45.6.974

Niederhoff J. (2007), Using separable programming to solve the multi constraints newsvendor problem and extension, European Journal of Operational Research, 176(2), 941-955. https://doi.org/10.1016/j.ejor.2005.09.046

Noori A.H., Keller G. (1986), One-period order quantity strategy with uncertain match between the amount received and quantity requisitioned, INFORM, 24(1), 1-11. https://doi.org/10.1080/03155986.1986.11731980

Ryu K., Yucesan E. (2010), A fuzzy newsvendor approach to supply chain coordination, European Journal of Operational Research, 200, 421-428. https://doi.org/10.1016/j.ejor.2009.01.011

Taleizadeh A. A., Barzinpour F., Wee H. M. (2010), Meta-heuristic algorithms for solving a fuzzy single-period problem, Mathematical and Computer Modelling, 54, 1273-1285. https://doi.org/10.1016/j.mcm.2011.03.038

Xu Y., Hu J. (2012), Random fuzzy demand newsboy problem, Physics Procedia, 25, 924-931. https://doi.org/10.1016/j.phpro.2012.03.179

Xu R., Zhai X. (2008), Optimal models for single-period supply chain problems with fuzzy demand, Information Sciences, 178, 3374-3381. https://doi.org/10.1016/j.ins.2008.05.012

Yao J. S., Chen M.S., Lu H. F. (2006), A fuzzy stochastic single-period model for cash management, European Journal of Operational Research, 170, 72-90. https://doi.org/10.1016/j.ejor.2004.06.017

Yager R.R. (1981), A procedure for ordering fuzzy subsets of the unit interval, Information Sciences, 24, 143-161. https://doi.org/10.1016/0020-0255(81)90017-7

Yu Y., Zhu J., Wang C. (2013), A newsvendor model with fuzzy price-dependent demand. Applied Mathematical Modelling, 37, 2644-2661. https://doi.org/10.1016/j.apm.2012.06.008

DOI: https://doi.org/10.6000/1929-7092.2017.06.34

(C) 2017 Natapat Areerakulkan; Licensee Lifescience Global.

This is an open access article licensed under the terms of the Creative Commons Attribution Non-Commercial License (http://creativecommons.org/licenses/by-nc/3.0/) which permits unrestricted, non-commercial use, distribution and reproduction in any medium, provided the work is properly cited. 